\title{
Qualidade da declaração de idade nos dados de óbito do Censo demográfico brasileiro de 2010
}

\section{Quality of age statement in 2010 brazilian demographic Census death data}

\author{
José Henrique Costa Monteiro-da-Silva' (D), Raphael Mendonça Guimarães² (D) \\ 'Centro Latino-americano e Caribenho de Demografia (CELADE), Comissão Econômica para a América Latina e o Caribe - \\ Santiago, Chile . \\ ${ }^{2}$ Fundação Oswaldo Cruz - Rio de Janeiro (RJ), Brasil.
}

Como citar: Monteiro-da-Silva JHC, Guimarães, RM. Qualidade da declaração de idade nos dados de óbito do Censo demográfico brasileiro de 2010. Cad Saúde Colet, 2021;29(esp.):205-210. https://doi.org/10.1590/1414-462X202199010452

\section{Resumo}

Introdução: O debate em torno do quesito de óbito no Censo Demográfico brasileiro foi retomado pela proximidade do Censo Demográfico de 2020. Há certa vantagem em obter informações de óbito por meio do Censo, mas é importante garantir a qualidade dessa informação. Objetivo: Analisar a qualidade da declaração de idade para os dados de óbito do Censo Demográfico de 2010. Método: Foram utilizadas as informações acerca dos óbitos nos domicílios, coletadas no questionário do universo do Censo Demográfico de 2010 do IBGE. A análise foi realizada a partir dos índices de Whipple e Myers. Resultados: Para o Brasil como um todo, os resultados observados atestam uma boa qualidade dos dados de declaração de idade dos óbitos. No entanto, quando é estratificada a análise ao âmbito de unidades federativas, algumas distorções foram verificadas, sobretudo nas unidades pertencentes às regiões Norte e Centro-Oeste. Conclusão: O uso dos dados de mortalidade para as análises em âmbito macro, do país como um todo, não requer ajustes para adequar as qualidades das informações, assim como para as análises em termos das grandes regiões. Palavras-chave: qualidade de dados; índice de Whipple; índice de Myers; mortalidade; censo demográfico.

\begin{abstract}
Background:The debate on deaths in the Brazilian population census has been resumed by the proximity of the next 2020 census. There are some advantages in obtaining death information through the census, but it is important to ensure the quality of this information. Objective: To analyze the quality of the age declaration for the 2010 demographic census death data. Method: Information on household deaths collected from the 2010 IBGE demographic census questionnaire was used. The analysis was performed from the Whipple and Myers indices. Results: For Brazil as a whole, the observed results attest to a good quality of the death declaration data. However, when the analysis at the state level is stratified, some distortions were found, especially in the North and Midwest areas. Conclusion: The use of mortality data for macro-level analyzes of the country as a whole does not require adjustments to suit the quality of the information, as well as for analysis in terms of large regions.
\end{abstract}

Keywords: data quality; Whipple index; Myers index; mortality; demographic census.

\section{INTRODUÇÃO}

Desde a década de 1990, a Organização das Nações Unidas (ONU) tem estimulado a inclusão de quesitos de óbito nos censos demográficos nacionais, sobretudo em países que apresentam sistemas de registro civil de baixa qualidade ${ }^{1,2}$. Recentemente, o debate em torno 
do quesito de óbito no Censo Demográfico brasileiro foi retomado pela proximidade do Censo Demográfico de 2020, diante dos cortes apresentados para o questionário, e pelas melhorias observadas na cobertura do registro civil no Brasil ao longo das últimas décadas ${ }^{2-4}$.

Queiroz e Sawyer ${ }^{5}$, ao analisarem os dados de óbitos nos últimos 12 meses dos domicílios brasileiros, encontraram resultados importantes e concluíram que: 1) apesar de apresentarem menor grau de cobertura do que as informações do Sistema de Informações Sobre Mortalidade do Ministério da Saúde (SIM/DATASUS), os dados de óbito apresentaram uma boa qualidade de cobertura; 2) os dados de mortalidade do Censo Demográfico permitem análises para subgrupos populacionais e de diferenciais socioeconômicos que são de difícil captação por parte dos dados de registro civil; e 3) os dados de mortalidade do Censo permitem calcular as taxas de mortalidade utilizando denominador e numerador de uma mesma fonte, contribuindo para uma melhor robustez das estimativas.

A declaração de idade em pesquisas domiciliares está suscetível a erros de preferência por dígitos, geralmente 0 ou 5, ou à falta de memória. Em geral, tais informações apresentam melhoria conforme a escolaridade da população ${ }^{6}$ e o modelo de coleta do dado: se for via declaração direta da idade por parte do entrevistado ou se for via declaração da data de nascimento ${ }^{7}$. Desta forma, o objetivo do presente estudo foi analisar a qualidade da declaração de idade para os dados de óbito do Censo Demográfico de 2010.

\section{MÉTODO}

Foram utilizadas as informações acerca dos óbitos nos domicílios, coletadas no questionário do universo do Censo Demográfico de 2010 do Instituto Brasileiro de Geografia e Estatística (IBGE) ${ }^{8}$. Nesta pesquisa, foram investigados o sexo, a idade e o mês de ocorrência dos óbitos ocorridos nos últimos 12 meses no domicílio. A análise foi realizada a partir dos índices de Whipple (para avaliar a atração por algum dígito específico, geralmente 0 ou 5) e de Myers (para avaliar o nível de atração por cada um dos 10 dígitos terminais - 0 a 9)7,9. Para efeitos de classificação, foram considerados os pontos de corte dos índices citados no Quadro 1.

O cálculo desses indicadores se deu a partir do pacote DemoTools, disponível no software $\mathrm{R}^{10}$.

\section{RESULTADOS}

O perfil etário dos óbitos coletados do Censo Demográfico de 2010 por idade simples já apresenta um cenário de possíveis distorções na declaração de idade (Figura 1). Notadamente, algumas regiões - Norte e Centro-Oeste - possuíam maiores distorções (dentes que se formam na pirâmide) que as demais a partir da visualização das pirâmides etárias de óbitos por idade simples. É importante mencionar que, para o país como um todo, essas distorções são suavizadas por conta do grande volume de observações.

A Tabela 1 mostra os resultados dos níveis de atração por dígito terminal do índice de Myers. Seguindo a linha do que se observou nas pirâmides etárias de óbitos, o país como

Quadro 1. Classificação de qualidade da declaração de idade pelo índice de Whipple e grau de atração pelo índice de Myers

\begin{tabular}{|c|c|c|c|}
\hline \multicolumn{2}{|c|}{ Índice de Whipple } & \multicolumn{2}{c|}{ Índice de Myers } \\
\hline Classificação & $\mathbf{I W}_{\mathbf{0}, \mathbf{5}}$ & Nível de atração & IM \\
\hline Preciso & $<105$ & Baixo & $0-4,9$ \\
\hline Pouco preciso & $105-109.9$ & Mediano & $5,0-14,9$ \\
\hline Aproximado & $110-124.9$ & Alto & $15,0-29,9$ \\
\hline Grosseiro & $125-174.9$ & Muito alto & $30,0-180,0$ \\
\hline Muito grosseiro & $>175$ & & \\
\hline
\end{tabular}



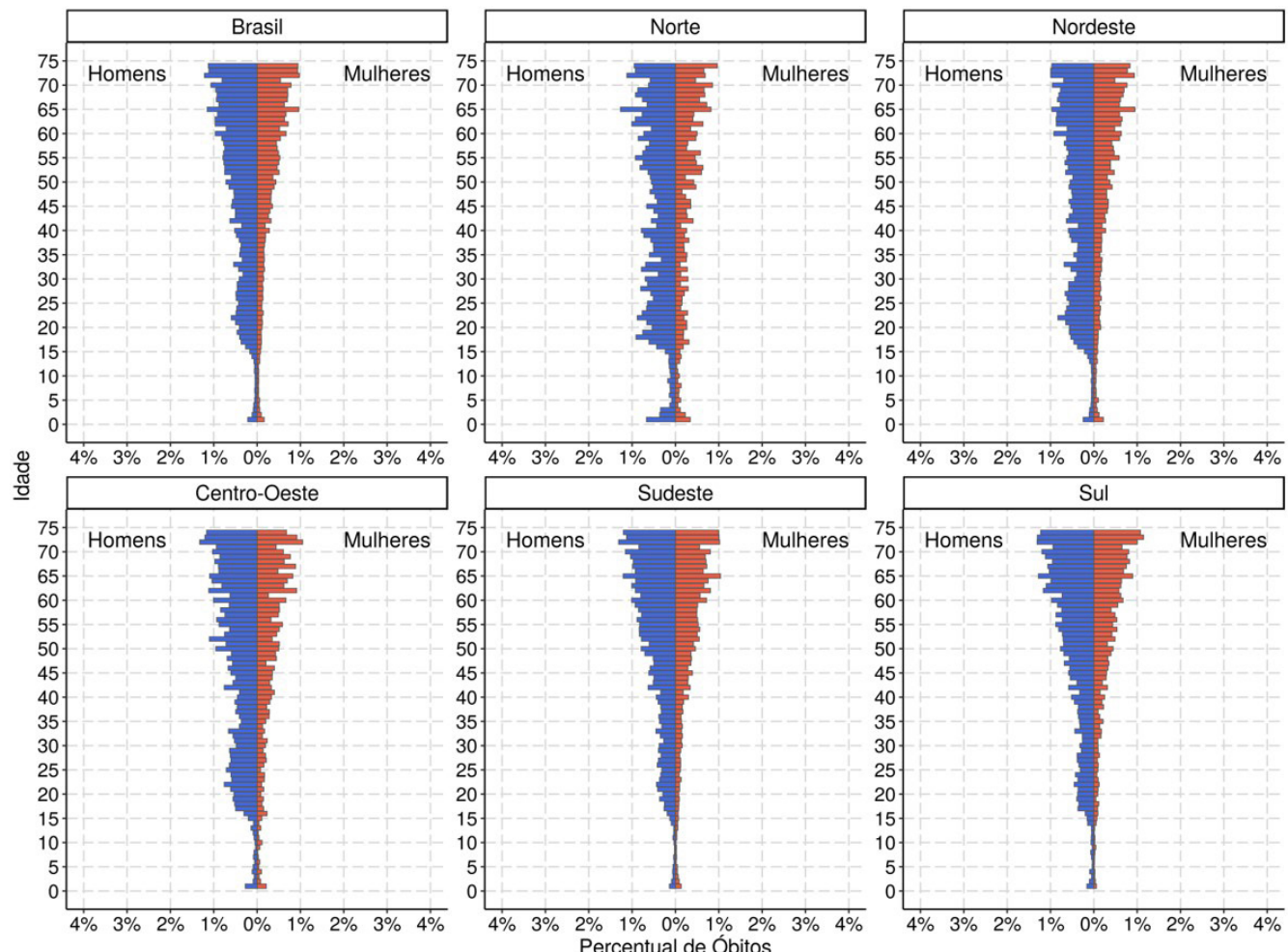

Figura 1. Pirâmide etária de óbitos, Brasil e grandes regiões, Censo Demográfico 2010

Tabela 1. Índices de Myers para idades declaradas de óbitos domiciliares. Brasil, grandes regiões e unidades federativas (UFs), 2010

\begin{tabular}{cccccc}
\hline UF & Homens & Mulheres & UF & Homens & Mulheres \\
\hline Norte & $\mathbf{5 , 8 3}$ & $\mathbf{6 , 3 2}$ & Nordeste & $\mathbf{3 , 0 4}$ & $\mathbf{4 , 2 5}$ \\
\hline RO & 7,26 & 10,72 & MA & 4,75 & 10,05 \\
\hline AC & 10,00 & 16,24 & PI & 4,81 & 6,08 \\
\hline AM & 8,57 & 13,10 & CE & 3,56 & 5,59 \\
\hline RR & 18,26 & 17,07 & RN & 9,45 & 11,27 \\
\hline PA & 6,48 & 6,9 & PB & 5,80 & 4,93 \\
\hline AP & 7,65 & 13,15 & PE & 3,67 & 7,00 \\
\hline TO & 6,29 & 11,77 & AL & 4,82 & 9,84 \\
\hline Centro-Oeste & 3,46 & 2,78 & SE & 7,25 & 9,31 \\
\hline MS & 5,91 & 7,01 & BA & 3,02 & 3,91 \\
\hline MT & 7,23 & 6,11 & Sudeste & 3,46 & 5,68 \\
\hline GO & 4,88 & 3,61 & MG & 3,62 & 8,76 \\
\hline DF & 10,72 & 7,25 & ES & 3,47 & 8,71 \\
\hline Sul & 2,98 & 2,91 & RJ & 4,56 & 6,15 \\
\hline PR & 3,97 & 3,70 & SP & 3,88 & 4,66 \\
\hline SC & 4,40 & 3,84 & & 3,19 & 4,49 \\
\hline RS & 3,23 & 3,67 & Brasil & & \\
\hline
\end{tabular}


um todo tem um baixo nível de atração digital, tanto para homens quanto para mulheres. No entanto, algumas diferenças regionais são notadas: as regiões Norte e Centro-Oeste apresentaram índices com nível de atração classificado como mediano ou alto para a maior parte de suas unidades federativas (UFs). Além disso, em geral, o índice de Myers apresentou maior nível de atração para a declaração de óbitos de mulheres do que de homens.

A Figura 2 apresenta as estimativas dos índices de Whipple para óbitos masculinos e femininos, sendo o índice para os homens indicado no eixo $x$ e o índice para as mulheres indicado no eixo y. É possível constatar que boa parte dos estados apresentava valores de Whipple considerados aproximados ou pouco precisos (entre 105 e 125). O país como um todo se encontrava em uma posição intermediária, com dados próximos de precisos para homens (aproximadamente 105) e pouco precisos/aproximados para mulheres (aproximadamente $110)$, assim como as suas grandes regiões.

Já na Figura 3, são apresentados os resultados dos cálculos do índice de Whipple para cada um dos dígitos terminais ( 0 a 9) para o Brasil (por região) e as grandes regiões (por UF). Claramente, quando são tomados grandes volumes de informações, como grandes regiões, os dados de declaração de idade do óbito apresentam menos valores pouco precisos do que quando a análise é estratificada por UF. Por fim, os resultados para os óbitos femininos indicaram informações de declaração de óbito de mais baixa qualidade entre as mulheres do que entre os homens.

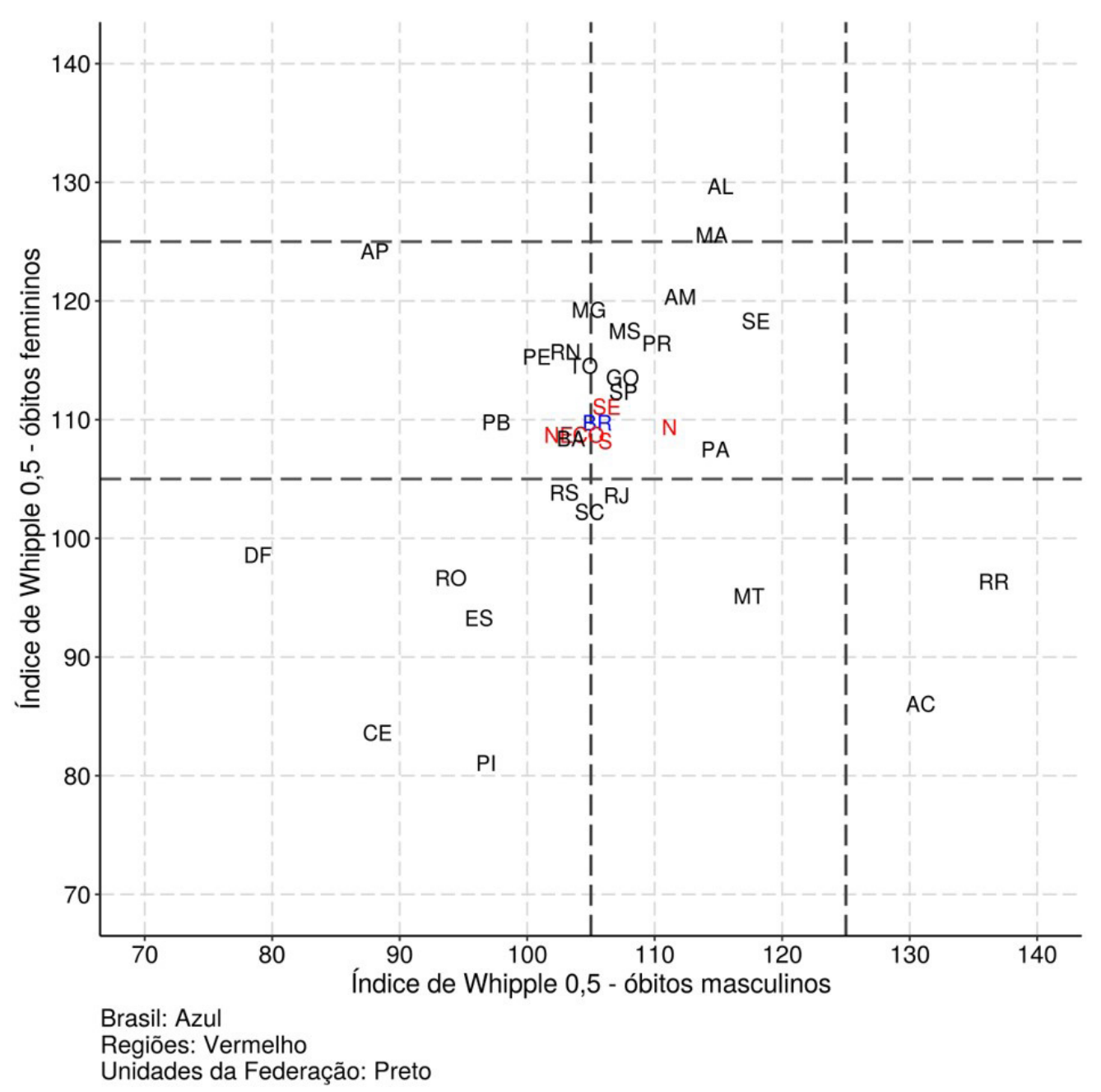

Figura 2. Índice de Whipple para óbitos masculinos versus femininos, Brasil e grandes regiões, Censo Demográfico 2010 


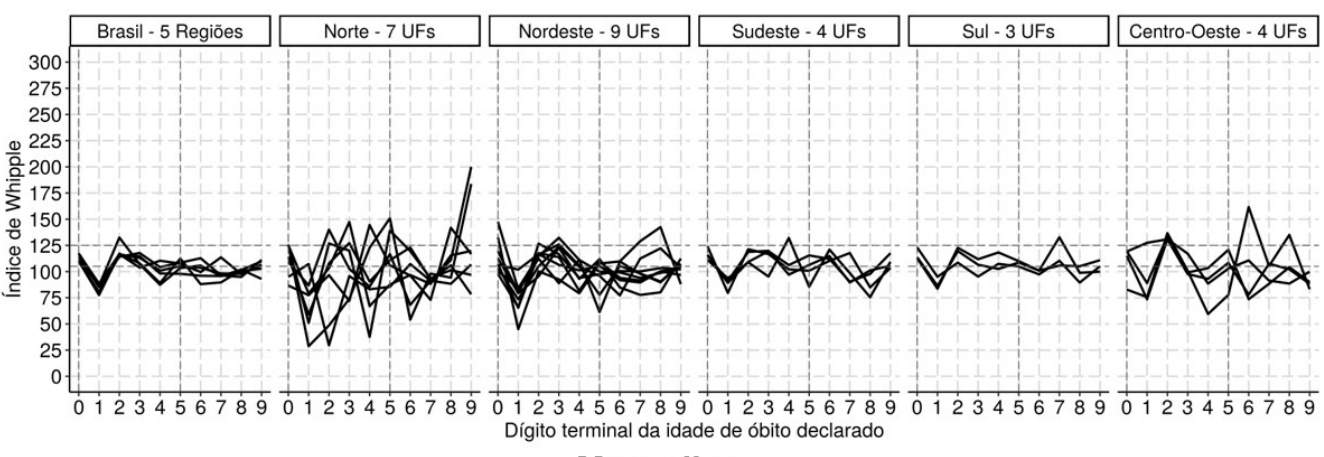

Masculino

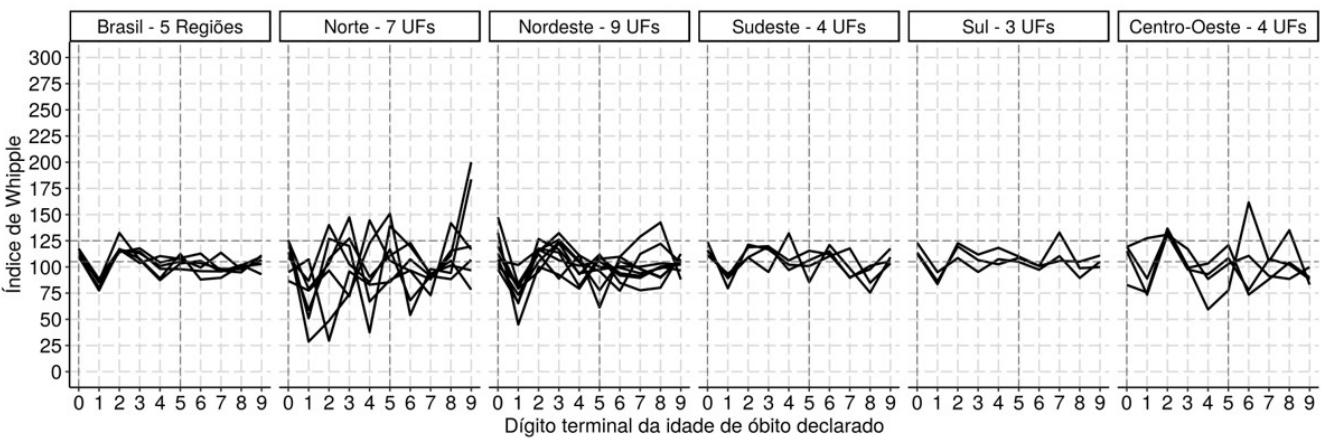

\section{Feminino}

Figura 3. Índice de Whipple por dígito terminal de idade de óbitos declarados segundo sexo, Brasil e unidades federativas (UFs), 2010

\section{DISCUSSÃO}

A redução no questionário do Censo Demográfico de 2020 fomentou uma série de discussões e debates acerca dos quesitos selecionados para retirada. A permanência do quesito referente aos óbitos no domicílio nos últimos 12 meses representou um alento para os demógrafos e pesquisadores da área da saúde. $O$ uso de tais dados em estudos de pequenas áreas e de populações tradicionais reitera a relevância desse quesito para os estudos de mortalidade e saúde da população².

Os dados de óbitos advindos dos Censos Demográficos demandam uma série de precauções e requerem correções de nível ${ }^{5}$, por conta da subenumeração que se deve à ocorrência de óbitos em domicílios monopessoais ou em casos em que o óbito resulta em dissolução do núcleo familiar. Ademais, o quesito de mortalidade está sujeito a erros de período de referência ou declaração de idade ${ }^{2}$, um tradicional problema das pesquisas domiciliares na demografia.

A declaração de idade ao óbito se coloca como um desafio a mais, pois depende do conhecimento correto da idade da pessoa falecida por parte do entrevistado, o que nem sempre acontece. Ainda assim, sabe-se que os dados do SIM/DATASUS possuem uma qualidade satisfatória de declaração de óbitos já há alguns anos, apresentando uma pequena vantagem para pessoas do sexo masculino com relação à atração por dígito" ${ }^{11}$. A avaliação da qualidade das informações de óbito se faz necessária, pois elas são amplamente utilizadas para estimativas nas áreas de saúde pública, epidemiologia e demografia que balizam uma série de políticas públicas de saúde.

Para o Brasil como um todo, os resultados observados atestam uma boa qualidade dos dados de declaração de idade dos óbitos. No entanto, quando é estratificada a análise ao âmbito de UF, algumas distorções foram verificadas, sobretudo nas unidades pertencentes às regiões 
Norte e Centro-Oeste. Os resultados dos índices de Myers e de Whipple corroboram o que se constata nas pirâmides etárias. Em geral, poucos estados apresentavam classificações de nível de preferência digital como sendo precisas ou com baixa atração, e na maioria a declaração de idade dos óbitos femininos possuía maior nível de atração do que os óbitos masculinos. No entanto, ao averiguar os resultados de Whipple por dígito terminal, foi notado um padrão de desigualdades regionais em termos de qualidade da informação de óbito. É possível dizer, portanto, que o uso dos dados de mortalidade para as análises em âmbito macro, do país como um todo, não requer ajustes para adequar as qualidades das informações, assim como para as análises em termos das grandes regiões. Porém, é recomendada a aplicação de métodos de interpolação, caso o objeto de estudo seja para as UFs, sobretudo se forem consideradas aquelas unidades pertencentes aos estados do Norte, Nordeste e Centro-Oeste.

\section{REFERÊNCIAS}

1. United Nations. Principles and recommendations for population and housing censuses. Nova York: United Nations; 2017.

2. Queiroz BL, Sacco N. ¿Es relevante incorporar la medición de la mortalidad en los censos de américa latina y el caribe? Rev Bras Estud Popul. 2018;35(2):1-8. http://dx.doi.org/10.20947/S0102-3098a0042.

3. Lima EE, Queiroz BL. Evolution of the deaths registry system in Brazil: associations with changes in the mortality profile, under-registration of death counts, and ill-defined causes of death. Cad Saúde Pública. 2014;30(8):1721-30. http://dx.doi.org/10.1590/0102-311X00131113. PMid:25210911.

4. Queiroz BL, Freire FHMA, Gonzaga MR, Lima EEC. Estimativas do grau de cobertura e da mortalidade adulta (45q15) para as unidades da federação no Brasil entre 1980 e 2010. Rev Bras Epidemiol. 2017;20(Suppl 1):21-33. http://dx.doi.org/10.1590/1980-5497201700050003.

5. Queiroz BL, Sawyer DOT. O que os dados de mortalidade do censo de 2010 podem nos dizer? Rev Bras Estud Popul. 2012;29(2):225-38. http://dx.doi.org/10.1590/S0102-30982012000200002.

6. Ewbank D. Age misreporting and age-selective underenumeration: sources, patterns, and consequences for demographic analysis. Washington, DC: National Academy Press; 1981.

7. Andrade PG, Pereira ACR, Camargo KCM, Brusse GPL, Guimarães RM. Calidad de la declaración de la edad de las personas mayores en países de América Latina y el Caribe: análisis de los censos demográficos de las décadas de 1960 a 2010. Notas Población. 2018;44(105):53-84. http://dx.doi.org/10.18356/e7a31a50es.

8. Instituto Brasileiro de Geografia e Estatística. Censo demográfico do Brasil 2010 [Internet]. 2010 [citado em 2019 Ago 15]. Disponível em: https://censo2010.ibge.gov.br/.

9. United Nations. Manual II: methods of appraisal of quality of basic data for population estimates. Nova York: Nações Unidas; 1955.

10. Riffe T, Fennell S, Aburto JM. DemoTools: Standardize, evaluate, and adjust demographic data [Internet]. 2019 [citado em 2019 Ago 20]. Disponível em: https://timriffe.github.io/DemoTools/.

11. Jacques FVL, Monteiro-da-Silva JHC, Guimarães RM. Qualidade da informação de idade nos registros de óbito no Brasil, 1996-2015. Rev Eletron Comun Inf Inov Saúde. 2019;13(1):158-171. 